\title{
Up Close and Personal: Social Presence in Mediated Personal Relationships
}

\author{
Daniel Gooch \\ Department of Computer Science \\ University of Bath \\ Bath \\ BA2 7AY \\ UK \\ D.J.Gooch@bath.ac.uk
}

\author{
Leon Watts \\ Department of Computer Science \\ University of Bath \\ Bath \\ BA2 7AY \\ UK \\ L.Watts@bath.ac.uk
}

\begin{abstract}
The likes of clarity and efficiency are good communication concepts for designers and evaluators of business communication tools. They make little sense, however, when the design context of an interactive system is the support of a personal relationship. What matters then is that people feel they are 'there' for one another. This paper describes a new way of understanding Social Presence in technologically mediated communication by relating it to a well-established psychological relationship construct: Closeness. We propose a model whereby an individual's long-term feeling of Closeness to others is influenced by communication events that are invested with a sense of Social Presence, as a function of the background level of psychological Closeness. Thus each communicative act, and its associated feeling of Social Presence, has an impact on the feeling of Closeness. We report a three-week-long study during which 18 participants reported daily ratings of Closeness, and communication-event ratings of both Closeness and Social Presence. Our findings are consistent with the model we propose, suggesting that systems for intimate relationships require consideration of both Social Presence and Closeness. We further consider methodological and measurement issues in the realm of personal relationships, and the expanding remit of $\mathrm{HCl}$ design as an active contributor to the world of experience and feelings.
\end{abstract}

Computer-Mediated Communication, Personal Relationships, Social Presence, Closeness, Intimacy

\section{INTRODUCTION}

As increasing numbers of relationships suffer through periods of spatial separation, so communication technologies assume greater significance for the people who are connected by them. Computermediated communication (CMC) technologies represent an important means through which partners, siblings, children and parents are able to express support for one another and sustain their relationships. Whilst it is possible to accomplish relationally meaningful acts through any communication medium, if better technologies are to be constructed with this type of communication in mind, it makes sense to consider how 'better' might be understood. In the 1970's, Short, Williams and Christie proposed Social Presence (SP) as a way of describing how media can influence feelings of connectedness (Short et. al, 1976). It remains as a key concept in CMC because it extends beyond matters of efficiency to include elements of communication experience. In this paper, we consider how SP has been operationalized and, more importantly, how it might be better used to inform researchers and designers of relational CMC technologies about how relational dynamics might condition the value of their innovations.

Short et al. (1976) defined SP as "the degree of salience of the other person in the interaction and the consequent salience of the interpersonal relationship" [p. 65]. SP is about surfacing the relationship in the mind of each party through the acts of communication they share. Another way to think of it is the level of emotional connectedness to have been engendered through a communicative act for each of the parties to it. SP is constructed in the mind of one party using the material representation of the other person through the medium, such as in the text of an email or a Facebook wall post. 
There has been considerable $\mathrm{HCl}$ design interest in the idea of communicating some form of personal connectedness through the use of innovative CMC prototypes (Kaye, 2006; Tollmar and Persson 2002; Tsujita et. al, 2008). These interests have translated into a variety of approaches towards the realisation of SP: Tollmar and Persson link it to physical presence and personal objects; Kaye to providing visible evidence of a distant loved one's thoughts, and Tsujita et. al to synchronising daily routines. Generally, such prototypes are vaguely grounded in the dynamics of an SP concept and, in consequence, have difficulties in formulating a clear evaluation strategy. One of the motivations of this paper is to create a better understanding of SP which will underpin the design and refinement of technologies to help support distance relationships.

It is convenient to treat $\mathrm{SP}$ as a relatively fixed property of the medium used to communicate. Short et al. are not wholly clear in their discussion of the concept, arguing variously that task context is a fundamental factor and yet that it is possible to rank media by a notional capacity for conveying SP. For example, that a phone conversation should always have greater potential to 'convey' SP than, an email. The SP-is-in-the-medium idea was consistent with Daft and Lengel's Media Richness Theory (richer permits more opportunity for SP transmission). However, a generalized sum-of-cues view cannot adequately account for relational phenomena that began to surface in the 1990's, such as hyperpersonal communication (Walther, 1996). As both the range of CMC opportunities have expanded and the degree to which $\mathrm{CMC}$ has become embedded in SP outside of work, so the relational significance of the 'tool-in-the-talk' has become ever more exposed. The familiarity of users with CMC systems is likely to govern their ability to see other people through the tools they use. Bradner and Mark, (2001) have presented work which showed equivalent levels of SP for people sharing video and application sharing. This suggests that SP is not purely a quality of the medium; rather it is a combination of the opportunity for people to personally manifest themselves to one another, and for others to read them. Opportunities to manage self-presentation and to reflect on the meaning of communication acts must also play into the SP equation. Hauber et. al, (2006) found that differences in spatiality in video conferencing led to differences in the level of SP. This is indicative that how the medium is used is significant, along with the choice of medium itself. Shih and Swan, (2005), investigating asynchronous online discussion tools, noted that the tone of communication affects feelings of SP, again indicating that it is not just a property of the communication medium but conditioned by the behaviour of actors and interpretations of recipients. Connell et. al, (2001) have demonstrated that when analysing telephone, face-to-face and Instant Messaging conversations for levels of SP, use of the telephone can generate greater feelings of SP than the other media. This is a result which is impossible to explain if SP is a mere quality of the communication medium: one would expect face-toface to be highest. Connell argues that SP must be formed to a level that is sufficient to complete the relevant task, suggesting that the task, relationship of participants and medium all contribute to the level of SP experienced by those who are involved.

From a design perspective, the issue at stake is how the medium is capable of contributing to the experience of SP in time. We wish to address questions that concern how a communication medium might factor into the instantaneous experience of SP, given an enduring 'task context' that is inherently longterm relational: maintaining a relationship when copresence is impractical or impossible. The complexity of the impact that media have on relationships might perhaps be better understood as a set of design properties of the communication system (e.g. asynchrony, pace, confidentiality, recordlessness), but such an idea could only be explored with better methods and measures of understanding the relational communication experience.

\section{MOTIVATION}

The motivation for the work we report here is primarily to improve on methods and measures for evaluating SP, with two concerns in mind. The first is that it is very difficult to compare results between research studies where differing instruments have been used, even if they claim to be measuring the same thing. The second is, as discussed below, SP as a measurement concept is both challenging and under specified. We hope our work will help other researchers, and ultimately designers, better reflect relevant aspects of the SP phenomenon.

$\mathrm{SP}$ is one of many psychological constructs that have been proposed to help explain an aspect of mental life: difficulties in conceptualizing and operationalizing such matters are to be expected. However, some such constructs have settled operationalizations in their research literature and so would address our first concern. Closeness is a relational construct which has been the focus of a great deal of research and measurement consideration (Agnew et al. 2004). "Closeness has generally been understood as what distinguishes among relationship categories, such as a close friend or parent versus a stranger" (Aron et. al, 1992). Closeness is a longer-term concept than SP. 
It can fluctuate within a broadly constant relationship category, or change between them - all friends, at some point, started off as being strangers; unfortunately, some will also finish up that way.

Operationalising SP in terms of Closeness has some advantages. Closeness is a well known concept which has immediate meaning to lay-people. It also has a more widely accepted definition than SP - a multidimensional construct consisting of the frequency of contact, the diversity of contact and the strength of contact (Aron et. al, 1992). We propose a new way of understanding SP. Our model takes the Closeness construct as a long-term relational concept against which SP should be understood. SP can be treated as a short-term feeling originating in particular communicative acts. We hypothesize that communicative acts impact on the longerterm feeling of Closeness. Communicative acts with high levels of SP increase the person's feelings of Closeness; conversely communicative acts with low levels of SP would decrease the person's feelings of Closeness.

To explain our model of SP, we will use the analogy of emotion and mood (Brave and Nass 2002). Emotions are object-directed; they imply and involve a person's reaction to a particular object or event. Moods are not directed at objects and tend to reflect a more generalized and persistent state of a person. An important difference is that emotions tend to be short-lived whereas moods are more enduring. The two concepts are interlinked; a person's mood biases the emotions they experience; a person's emotions contribute to the mood they are in (Frijda, 1994). In this analogy, SP functions in a manner similar to emotion, in that it is triggered by the way the other person is revealed in the communicative act. Closeness functions in manner similar to mood because it represents a generalized and relatively persistent sense of well-being within a relationship.

There are two main reasons why it is worth defining $\mathrm{SP}$ in this way.

The first is that the term SP has been used by researchers for years to connote a property of the medium, that a phone call will always have a certain level of SP and an email will have a different level. As we have previously discussed, such a conception is no longer tenable in the face of the evidence. As such, a clearer and more robust understanding of SP needs to be developed. The second is the lack of a commonly accepted measure: it is very difficult to compare results between research studies if they have operationalized the concept in different ways; each could be exposing different facets of the intended phenomenon, i.e. SP, mixed in with other matters that simply cannot transcend the technical context of the studies.

There is no shortage of methods for measuring SP, including at least 12 questionnaires (Van Baren and IJsselsteijn, 2004). The issue is more complex than a mere absence of measures. Not all of the questionnaire details are published, making it hard for other researchers to use the same questionnaires. Most suffer from reliability and/or construct validity issues. There are also pragmatic issues with consequences for their value as experiential measurement: some extremely long (Networked Minds (Biocca, Harms and Gregg 2001) contains 40 questions) or so focussed on the particularities of a specific technology they are not transferable. It is therefore hard to use these measures in an experiential evaluation and be confident in the data they return.

Given this variety, the two most commonly used questionnaires are Short et al.'s Semantic Differentials and Biocca's Networked Minds. We will use these as case studies to consider the weaknesses of evaluation tools currently available.

The Networked Minds measure a carefully constructed questionnaire which asks 40 questions, each related to eight sub-scales believed to contribute to SP, each scored on a Likert Scale. Networked Minds was checked for consistency by undertaking a comparative task carried out by a large number of participants. The results showed that face-to-face was more socially present than a teleconferencing system. If this hypothesis is accepted then this provides some evidence of validity. Regardless of the support for the hypothesis, the scale has not been validated for use with any other medium.

Semantic Differentials is intended to measure a medium's ability to support SP. The questionnaire asks participants to rank the medium in terms of two diametrically opposed adjectives (e.g. impersonal vs. personal). The first criticism of this measurement is the fact that it asks participants to provide a measure of the medium, not of their experience of the presence of the other person during conversation. The second criticism is the lack of evidence for using it as a measure of SP. The Semantic Differentials method was tested for reliability by using it with a large number of participants and then statistical testing for consistency but no other form of validation was undertaken.

Our primary research question pertains to whether $\mathrm{SP}$ measure can be systematically related to a valid and robust instrument for measuring psychological Closeness: the Inclusion of Other in Self (IOS) scale (Agnew et al. 2004). Our secondary question 
is whether, given a relational 'task context' and baseline Closeness measure, we are able to contrast the experience of SP in conversation through CMC technologies in a more discriminating manner than has previously been attempted.

\section{METHOD}

Our research questions refer to a sphere of human activity wherein experimental investigation has little face value. There is so much vital relational context surrounding each communication act that isolating them in a lab setting is unlikely to be revealing. It would probably be both too artificial to reflect the established communication practices of people in relationships, and artefactual, in terms of introducing unwanted influences on actions and reactions due to the unfamiliarity of the setting. Additionally, we are operating in a world of experience and feelings, a world which cannot be directly observed and so is not best suited to methods that emphasize controlled observation. Consequently, our investigation of SP phenomena depends on access to behaviour, attitudes and experiences in 'real-world' settings.

We also wanted to investigate changes in Closeness over time, since Closeness is conceptualized as an inherently dynamic but slow-changing feeling. As such, we adopted a periodic self-report method. Each participant was asked to keep two types of record, one using a periodic and the other an event-based sampling method. The periodic record required participants to complete a daily measure of Closeness, using the IOS scale (see (Aron et. al, 1992) and (Agnew et al. 2004)). This operates in a manner akin to a graphical Likert scale, in that participants are asked to express their reaction to a question on a seven-point scale but each point on the scale is represented by an image rather than a number in a linear series. The question in IOS is 'Please circle the picture below which best describes your relationship with your [romantic] partner'. IOS represents points on this scale as seven pairs of circles, each labelled 'self' and 'other'. At one extreme - corresponding to 'not at all close' - shows self and other as two circles that abut but do not intersect. The other extreme shows as almost complete overlap, the non-intersecting portions thus representing only a small fraction of the individual selves preserved outside of the relationship. Points in between thus vary in the degree to which respondents are able to express their relationship in terms of the proportion of themselves that is comprised of the other. As a graphical rather than text-based scale, it reflects similar concerns to those that motivated the development of the Self Assessment Manikin to evaluate affective experience (Bradley and Lang, 2002).

The event-based record was completed each time the participant had communication with their partner - this was called the contact diary. The contact entries recorded basic information about the communication act including time of contact, length and method of communication and who initiated/ended the contact. In addition, participants were asked to complete two questionnaires, one being the 9 item SP questionnaire developed by (Short et. al, 1976), and the other being the IOS Closeness measure, as described above. Short et al.'s original concept and 9 item questionnaire was developed almost exclusively from, and intended for, use in time-limited laboratory-based studies but we felt it important to be able to relate our work to the longest-established variant of SP measure. Participants were asked to keep continuous records for a period of three weeks.

Participants were recruited through emails and posters on a British university campus. Participants were encouraged to recruit their partners to undertake the study. The types of relationships in our study thus included lovers, parent/child and sibling relationships. Participants were asked to keep records of their personal communications and feelings for three weeks. The daily diary was to be completed every morning; the contact diary as soon after the contact as possible. 38 participants were originally recruited for the study. Eight of these decided not to complete their diaries, reporting that they found the study too personal in the end to share. A further six said that they had forgotten about it and we had no response from the remaining six. Only 18 returned a complete set of records over the whole study period ( 11 men and 7 women), indicating that they were sufficiently motivated to complete the study without being overly concerned about us seeing what they had written.

\begin{tabular}{|c|c|}
\hline Relationship Type & Number of Participants \\
\hline Partner & 10 \\
\hline Friend & 3 \\
\hline Mother & 1 \\
\hline Sister & 1 \\
\hline Son & 1 \\
\hline Work Friend & 1 \\
\hline Brother & 1 \\
\hline
\end{tabular}

Table 1: Number of participants recording communication for each relationship type

The quality of the data collected with longitudinal self-report studies must always be a concern. People may/can self censor, confabulate and forget or miss 
relevant episodes. The risk of these was minimised by making the diary entries as short as possible such that the effort required to fill them in was minimised. Beyond that, the limitations of the method must be accepted and acknowledged. We present our the data as a reasonable reflection of our participants' communication practices and experiences whilst conceding that some self censorship was possible for at least some of our participants.

\section{ANALYSIS AND RESULTS}

All participants filled in all of their daily entries giving 378 ratings of Closeness (18 participants $\times 21$ daily records). The number of contact entries varied dramatically between participants. There were 357 contact entries in total, the mean was 19.8, SD was 10.7 , median was 19 . The maximum for any individual was 37 entries, the minimum was 5 .

\subsection{Does SP in Communication Acts Influence Daily Feelings of Closeness?}

Our first question is whether the event-based (contact) ratings of Closeness and SP had a consistent relationship with the periodic (daily) rating of Closeness (i.e. the longer-term feeling of Closeness). We thus grouped contact ratings into the 24 hour period preceding each daily rating. Arranging the data in this manner allows us to compare contact SP and Closeness ratings against the change in Closeness over time. The result of such an analysis should shed light on the high-level idea in our model, that contact experiences function as a delta-change driver on the longer term feeling of Closeness.

Our model describes a causal relationship between SP experienced during a communication act and the more enduring feeling of Closeness. We do not argue that this is a uniquely causal relationship: it is clear that Closeness between people can be affected by other matters besides direct communication (e.g. hearing of a partner's infidelity or kindness through a friend, discovering theft etc). Nevertheless, we argue that, if contact SP ratings within our defined 24 hour period are low, subsequent daily Closeness ratings would also be low. Similarly, if contact SP ratings are high, we believed that daily Closeness ratings would also be high. We had no expectations about mid-level contact ratings or daily ratings that did not change (i.e. are 'maintained' from day to day) but wanted to understand how, over time, how associations with them might reveal something of the maintenance dynamic in personal relationships mediated through technologies.

The Closeness contact ratings are being gathered for a methodological purpose rather than for a conceptual purpose. There are two things to consider. The first is that we are not arguing that $\mathrm{SP}$ is the same as Closeness. By gathering both contact SP and Closeness data, we can empirically investigate their relationship in terms of similarities and differences. What we are trying to do is investigate the link between SP and Closeness at a conceptual level by exposing how people express their reactions to communication contexts in terms of their ratings in and around the moment of the communication act. The IOS measure has been taken to reflect a range of relational issues: we use it here as a lens on SP. We have already discussed how weak the SP measures are in terms of validation. In methodological terms, by gathering the contact Closeness ratings, as well as daily Closeness ratings, we are able to relate SP ratings to the more robust IOS Closeness ratings.

The data we gathered does not conform to the normal distribution and is not interval in nature, thus we sought a nonparametric statistical treatment that would permit us to address these issues. For comparisons between groups, we used the Kruskal-Wallis mean ranks test and, for correlations, Spearman's rank test.

\subsubsection{Relating Contact Ratings to Change in Daily Closeness}

Our model of SP is that it impacts the longer-term feeling of Closeness. To test this idea, we began by considering the change in our daily Closeness ratings from day-to-day. All diary entries were filled in at the beginning of the day. Therefore all acts of communication within that day can be predicted to have had an impact on the next rating of Closeness. We do not have a large enough data set to consider every possible change in Closeness - there are 6 factorial different increases (and subsequently 6 factorial decreases) which, along with 7 maintenance levels, gives 1,447 different ways of categorising the change in Closeness between two given days. By grouping the change in daily Closeness as a 'decrease', 'maintenance' or 'increase' of Closeness, we can then associate the contact SP and Closeness scores with that change in daily Closeness. Our decision to use a conservative non-parametric approach is consistent with ordinal data of this type. We might then be able to see if levels of contact SP and Closeness were consistent with these shifts in daily Closeness.

The mean rank of Contact SP scores differed significantly as a function of change in daily Closeness ratings $(H(2)=15.757, P<0.001)$. Contact SP scores were ranked marginally lower on days when Closeness ratings decreased and higher when daily Closeness ratings increased. Surprisingly, however, on this analysis the highest 


\begin{tabular}{|c|c|c|c|}
\hline $\begin{array}{c}\text { Change in } \\
\text { Daily } \\
\text { Closeness }\end{array}$ & $\mathrm{N}$ & $\begin{array}{c}\text { Mean Rank of } \\
\text { Contact Social } \\
\text { Presence Score }\end{array}$ & $\begin{array}{c}\text { Mean Rank of } \\
\text { Contact } \\
\text { Closeness } \\
\text { Score }\end{array}$ \\
\hline Decrease & 82 & 140.15 & 147.88 \\
\hline Maintained & 165 & 188.07 & 186.54 \\
\hline Increase & 87 & 154.26 & 149.89 \\
\hline Total & 334 & & \\
\hline
\end{tabular}

Table 2: Mean rank of contact Social Presence and Closeness scores for each category of change in daily Closeness rating

ranked SP scores were associated with daily Closeness ratings that did not change (see table 2: 'Maintenance').

A similar pattern is evident in the contact Closeness ratings. Again, differences in mean ranks are statistically significant $(\mathrm{H}(2)=13.478, \mathrm{P}=0.001)$ but the effect is clearly dominated by higher ranked scores for maintenance of the daily Closeness rating. On this measure, changes are indistinguishable.

Our primary expectation was that there was a relationship between contact SP and daily Closeness. This was initially analysed using the change in rating rather than focussing on the absolute value of the daily Closeness rating. Thus a change from 1 to 2 in Closeness rating was rated as 'Up' whereas maintaining a Closeness rating of 6 was regarded as 'Same'. Looking at the raw data in detail, there were 3 incidents of maintenance at a low level, 60 at a medium level and 68 at a high level. It could be that our participants' relationships were too uniformly strong to permit expressing effects based on deltas from a low baseline. In other words, 'maintained' seems to have both the majority of 'high' SP ratings and the majority of enduringly 'good relationships', possibly confounding the two in this analysis. It is also indicative of possible ceiling or floor effects here.

\subsubsection{Relating Contact Ratings to Levels of Daily Closeness}

To address the issues with our first, change-based analysis, we subjected our data to a second analysis. The data was grouped by daily ratings as being high (rating of 6-7), medium (rating of 3-5) and low (rating of 1-2). We then associated each daily Closeness grouping with contact SP and Closeness ratings (so each rating belonged to a daily category) and ranked them. As before, the mean rank scores were calculated for contact SP and Closeness ratings to see how the magnitude of contact Closeness and SP scores were associated with the daily level of Closeness. If there was no relationship between contact SP and daily Closeness, there would be no difference between the mean rank SP scores as a function of their daily Closeness grouping (i.e. it would be revealed as a random association).

\begin{tabular}{|c|c|c|}
\hline $\begin{array}{c}\text { Level of } \\
\text { Daily Closeness }\end{array}$ & $\mathrm{N}$ & $\begin{array}{c}\text { Mean Rank of Contact } \\
\text { Social Presence Score }\end{array}$ \\
\hline Low & 11 & 118.73 \\
\hline Medium & 185 & 140.38 \\
\hline High & 138 & 207.75 \\
\hline Total & 334 & \\
\hline
\end{tabular}

Table 3: Mean rank of contact Social Presence scores for each category of daily Closeness rating

This analysis shows a clear picture, both in terms of our expectations and by discriminating among the mean ranked contact ratings more categorically than before. Contact SP scores are associated with low (below average) daily Closeness ratings are lowest ranked, contact SP scores in the medium daily Closeness group are approx. 20 rank places higher, and contact SP scores in the high daily Closeness group are a further 60 rank places higher again. These differences are statistically significant $(\mathrm{H}(2)=41.533, \mathrm{P}<0.001)$ (see table 3 ). These ranked ratings conform with our expectations about the association between contact SP and daily Closeness. It is notable that the mean rank difference between the low and medium daily Closeness groups is considerably overshadowed by the difference between the medium and high groups.

\begin{tabular}{|c|c|c|}
\hline $\begin{array}{c}\text { Level of } \\
\text { Daily Closeness }\end{array}$ & $\mathrm{N}$ & $\begin{array}{c}\text { Mean Rank of Contact } \\
\text { Closeness Score }\end{array}$ \\
\hline Low & 11 & 71.55 \\
\hline Medium & 185 & 134.44 \\
\hline High & 138 & 219.47 \\
\hline Total & 334 & \\
\hline
\end{tabular}

Table 4: Mean rank of contact Closeness scores for each category of daily Closeness rating

Contact Closeness reflects the same general pattern (see table 4): ratings of contact Closeness associated with low daily Closeness are ranked lowest, contact Closeness in the medium daily Closeness group are mid-ranked, and contact Closeness in the high daily Closeness group rank highest of all $(\mathrm{H}(2)=77.006, \mathrm{P}<0.001)$. Unlike our contact SP analysis, the mean rank position of low daily Closeness ratings is much lower than our medium group of daily Closeness; at approximately half the mean-rank position. The rank orders for medium and high contact Closeness are very similar to pattern evident in for contact SP scores. 


\subsection{Relating Closeness and Social Presence}

We are also interested in whether we can use the Closeness measures as an indication of SP. As such we looked for a correlation between the contact diary ratings of SP and Closeness.

Spearman's rho shows a significant positive correlation between SP scores and Closeness scores $(r$ $=0.728, n=357, p<0.001$ ). Higher ratings of Closeness were correlated with higher ratings of SP but, as the differences in our foregoing mean-ranks analysis suggests, they are not identical.

Previous work has indicated that SP is impacted by medium (e.g.. (Connell et. al, 2001), (Short et. al, 1976)). As such we have compared the contact SP data by medium (see table 5) and contact Closeness by medium (see table 6 ).

\begin{tabular}{|c|c|c|}
\hline Medium & $\mathrm{N}$ & $\begin{array}{c}\text { Mean Rank of Contact } \\
\text { Social Presence Score }\end{array}$ \\
\hline Face-to-Face & 81 & 250.43 \\
\hline Skype & 62 & 226.23 \\
\hline Telephone & 78 & 161.39 \\
\hline IM & 20 & 136.60 \\
\hline SMS & 85 & 135.11 \\
\hline Email & 23 & 94.33 \\
\hline Facebook Wall & 8 & 77.19 \\
\hline Total & 357 & \\
\hline
\end{tabular}

Table 5: Mean rank of contact SP scores by CMC

There was a statistically significant difference between the contact SP scores as a function of contact medium $(\mathrm{H}(2)=96.393, \mathrm{P}<0.001)$. As prior investigators have found, Face-to-Face communication is associated with the highest levels of SP. We find mean-ranked contact SP scores for IM and SMS to be indistinguishable - also unsurprising in the light of prior work. Our data shows that Skype (VOIP) leads Telephone communication by 60 rank places. We are able to report that Facebook wall postings (among people in close relationships) are associated with the lowest levels - a communication context not previously considered in SP research.

There are also significant differences between contact Closeness ratings when ranked by medium $(H(2)=94.438, P<0.001)$ but the pattern of rank positions is not the same as for contact SP. Faceto-Face tops the ranking, again unsurprisingly, and Facebook wall posts are also at the bottom of the list - they did not make our participants feel at all close to one another. However, our Closeness ratings make it difficult to distinguish Skype and Telephone - our participants felt much more Socially Present in Skype than on the phone, but no Closer to one another. The reverse is true when we consider

\begin{tabular}{|c|c|c|}
\hline Medium & $\mathrm{N}$ & $\begin{array}{c}\text { Mean Rank of Contact } \\
\text { Closeness Score }\end{array}$ \\
\hline Face-to-Face & 81 & 262.07 \\
\hline Skype & 62 & 178.48 \\
\hline Telephone & 78 & 171.42 \\
\hline SMS & 85 & 155.35 \\
\hline Email & 23 & 111.54 \\
\hline IM & 20 & 94.15 \\
\hline Facebook Wall & 8 & 73.19 \\
\hline Total & 357 & \\
\hline
\end{tabular}

Table 6: Mean rank of contact Closeness scores by CMC

SMS and IM on both measures. Unlike the picture we see with our SP scores, the mean SMS and IM rankings for Closeness are separated by 60 rank positions: our participants felt considerably closer to one another when they texted than when they IM'd, though they felt equal SP. These contrasts matter to the extent that they provide an empirical basis for improving our understanding of SP in the context of communication between people who care about one another.

\section{DISCUSSION}

We hypothesised that, for people in personal relationships, communicative acts have an impact on long-term feelings of Closeness via the degree to which each individual experiences the feeling that the other party is Socially Present to them. We tested this hypothesis by first looking at the association between contact ratings (of SP and Closeness) and the change in Closeness ratings between days. We found both contact SP and contact Closeness ratings were ranked lowest for days when there was a decrease in Closeness, and were midranked for days when there was an increase in daily Closeness ratings. Our hypothesised model, whereby positive SP experiences should positively influence feelings of Closeness, predicted that highest-ranked SP scores should be associated with days when Closeness increased. However, maintenance of the daily Closeness level was associated with the highest-ranked Closeness ratings of all. Maintenance of a daily Closeness level does not take into account whether the maintenance is of a high level (e.g. Closeness rating of 6 to 7) or a low level (Closeness rating of 1 to 2). So, as well as comparing the contact data against changes in daily Closeness, we compared these data against the level of daily Closeness ratings for the 24 hour period to which they applied (i.e. the day after the communicative act had occurred). Tables 3 and 4 show the results. These match our predictions: low-, medium- and high-ratings of daily Closeness 
correspond respectively to low-, medium- and highranked communicative acts on SP and Closeness.

We argue that our data supports the proposition that SP can be treated as influential on long-term Closeness based on the net effect of communicative acts. Our data strongly support the association between SP experiences in communication and daily feelings of Closeness. They do not, however, demonstrate a causal connection. We previously used the analogy of emotion and mood to try to explain the mechanism by which we argue $\mathrm{SP}$ relates to Closeness. We believe that each communicative act, treated in our analysis as some episode such a phone call or receipt of an email, has an impact on a person's understanding of the state of their relationship with the other.

In addition to the positive aspects of communication on Closeness, we must also consider what happens when there is a lack of communication. Tables 7 and 8 combine our interest in change and absolute level of daily Closeness ratings to consider how frequency operates. Table 7 compares the number of days without communication and Table 8 contrasts the frequency of days when our participants got in touch with one another by some means.

\begin{tabular}{|c|c|c|c|c|c|}
\hline & Down & Same & Up & Total & Percent \\
\hline Low & 8 & 5 & 3 & 16 & 14 \\
\hline Mid & 22 & 30 & 20 & 72 & 65 \\
\hline High & 0 & 14 & 9 & 23 & 21 \\
\hline Total & 30 & 49 & 32 & & \\
\hline Percent & 27 & 44 & 29 & & \\
\hline
\end{tabular}

Table 7: Distribution of communication-free days by level of daily Closeness rating

\begin{tabular}{|c|c|c|c|c|c|}
\hline & Down & Same & Up & Total & Percent \\
\hline Low & 7 & 3 & 0 & 10 & 4 \\
\hline Mid & 44 & 60 & 23 & 127 & 50.8 \\
\hline High & 9 & 68 & 36 & 113 & 45.2 \\
\hline Total & 60 & 131 & 59 & & \\
\hline Percent & 24 & 52.4 & 23.6 & & \\
\hline
\end{tabular}

Table 8: Distribution of days with communicative acts by level of daily Closeness rating

The tables should be read as follows. Each cell represents the number of days with or without a communicative act which fall into a given daily Closeness category. Columns organise the data by change, showing whether Closeness decreased, was maintained, or increased compared to the previous day. Rows contrast the same data by whether the daily Closeness was rated at a low, medium or high level.
Given the difference in population size, we must consider the percentages for each category. Looking at the relative changes in Closeness, there seem to be no major differences between the distribution of days across decrease, maintenance or increase in Closeness between days with communication and days without. It seems that changes in levels of daily Closeness, once again, are not informative.

However, considering the absolute values, we can see a clear difference. Days with low levels of daily Closeness are more common on days with no communication. Days with medium levels of Closeness are more common on days with no communication. Days with high levels of Closeness are less common on days with no communication. This fits well with the proposed model. There are more contactless days with low/medium levels of Closeness compared to days with communication. It could be that the absence of communication will mean that the lack of SP figures in our participants' understanding of their relationship, and may be equivalent to a low ranked score. The lower number of contactless days that were rated with high levels of Closeness, compared to high-rated days with communication, is consistent with this idea. The model thus fits with regards to the benefits of high SP and the negatives of an absence of SP. The distribution of communicative acts, as well as the level of SP ratings, leading up to each daily Closeness rating, suggest that frequency and level work together. The manner in which they are combined is probably not simply additive (one can have too much of a good thing) but further research is needed.

We found that both SP and Closeness vary depending on the medium used to communicate. This is noteworthy for a number of reasons. The result demonstrates that the medium people use to communicate has an impact on their feelings of both SP and Closeness, though not to the same extent and in a way that seems to differ by medium. This suggests a valuable conceptual and methodological link between SP and Closeness. Looking at the medium data, most of the ranks are similar between SP and Closeness with the exception of Skype and IM. Although the ordering of the mediums between SP and Closeness are different, there are roughly three groupings within each measure: for SP Face-to-Face and Skype; Telephone, IM and SMS; Email and Facebook Wall. For Closeness - Face-toFace, Skype, Telephone and SMS; Email; IM and Facebook Wall.

We took the unit of analysis as the records of each individual. Most but not all of our completed records were returned by pairs of people. We were 
concerned with individual feelings and experiences of social presence and closeness, as reflections of an individual's emotional state, in developing a conjoint approach to assessing instantaneous and enduring feelings. However, it is possible that this decision may have influenced the rankings of some $\mathrm{CMC}$ technologies. For evaluations of a specific technology, it would be more valid to treat the records of dyads as the unit of analysis.

In conjunction with this disparity in results, we have the correlation data to contend with. There was a strong general correlation between contact SP and contact Closeness ratings. We see two explanations for why this may be the case. Our motivation for this work is in part to develop relevant methodological approaches for designing and evaluating relational technologies. So the first possibility must be that the two measures (SP and Closeness) are essentially measuring the same thing and that our contrasting results for CMC media are artefacts of our method, perhaps due to the way our participants interpreted our instructions on the meaning of each measure with respect to their communicative acts. We see this as a weak criticism but open to others to challenge with their own data and procedures for its collection.

The second explanation is more complex. The two concepts are clearly similar, as demonstrated throughout the various tests we have presented and the strong correlation. However, we argue that, in our relational context, the CMC media contrasted by our measures imply substantive differences between SP and Closeness. The question such a proposition raises then is how one might enumerate the properties of CMC media that play into personal relationships. We should also consider more deeply what our relational context implies about the 'conversational work' that is performed through communicative acts. CMC experiments typically set up a conversation before hand and then see how it plays out. We did not do this and so the content of mediated conversations in our study must have been far more variable than experimental researchers have previously allowed. At some level, our participants may have decided not to choose a notionally 'high presence' communication context to talk about things that they felt needed high presence. There is likely to be a certain level of pre-meditation and effort which has been invested into the communication before it has even occurred. There is some work (e.g. (Gooch and Watts 2011), (Riche et. al, 2010)) which indicate that the level of percieved effort has a positive effect on SP. This would account for the higher levels of SP over Closeness for IM and Skype conversations. We see this in itself as an important matter since we are trying to understand how SP works through the attitudes, words and deeds of those who participate in our research. In other words, their choices are material to our research.

We started this paper by discussing some difficulties in researching SP. One of these is the lack of grounding for a measure of SP in a relational context. Although we have not directly investigated the use of new measurement techniques, the results we have found indicate that the diaries we have used could be used in other studies looking at SP (e.g. the continuation of the Magic Sock Drawer Project, (Gooch and Watts 2011).

\section{FURTHER WORK}

Although we have found some evidence to support our model linking SP to Closeness, there are a number of areas of further work which we would like to investigate in order to better understand the model. The first is related to our previous analogy of emotion. Emotion is generally described in terms of valence (i.e. positive or negative) and intensity (i.e. strength of feeling). Thus far we have treated $\mathrm{SP}$ in the same was as prior researchers - as a single dimension akin to intensity. However, this runs the risk of treating low intensity as bad and high as good for personal communication. What is unclear and not understood is what form valence of SP would take, if any. For example, a lover's tiff might be of high SP intensity but very negative at the time, though perhaps setting a context for restoring positive relations (Billings and Watts, 2010). Would the protagonists simply experience low SP? Or is there some kind of negative or inverse SP that would take this into account? These questions are vital if the nature of relational communication through mediating technologies is to be understood.

We wish to broaden and deepen our treatment of empirical data, especially to understand how frequency interacts with the intensity of SP experiences. Although the 378 days of largely quantitative data furnished by our 18 participants gives us some useful insights into the potential of our model, we are engaged in other design-focused activities with a richer set of qualitative data to test and refine our ideas. Much can be learned from qualitatively investigating how significance is attributed to aspects of communication within a particular technological environment to complement the approach we have taken in this paper. For example, research on intimacy in World of Warcraft (Pace, Bardzell and Bardzell, 2010). More data of this nature would also help us to understand how other factors impact the model. There are a number of factors we have identified as being worth investigating. Connell et. al, (2001) have previously 
identified task, medium and relationship as being significant when considered SP. As such, these need to be considered with regards to our model. Further data would help to determine how relationship and medium impact on the model including to unpack the relational work conducted in close personal conversations. This is necessary if the full story about the interaction between SP and Closeness is to be told, and indeed how these concepts can give researchers and designers purchase on interpersonal relationships as they are enacted through CMC technologies. In this way, we feel the model we propose can serve to better understand SP such that we can design communication technologies to better support people's relationships.

\section{REFERENCES}

Aron, A., Aron, E. and Smollan, D., Inclusion of Other in the Self Scale and the Structure of Interpersonal Closeness. Jour. of Personality and Social Psychological Association, 63(4), 1992. pp. 596-612.

Agnew, C.R., Loving, T.J., Le, B. and Goodfriend, W. 'Thinking close: Measuring relational closeness as perceived self-other inclusion' In Handbook of Closeness and Intimacy. pp. 103-115. Lawrence Erlbaum Associates, 2004.

Billings, M. \& Watts, L. (2007): A Safe Space to Vent: Conciliation and Conflict in Distributed Teams. 10th European Conference on Computer Supported Cooperative Work, Limerick, Ireland, 24 - 28 September 2007. pp. 139-158.

Biocca, F., Harms, C. and Burgoon, J.K. Toward a more robust theory and measure of social presence: Review and suggested criteria. Presence, 12 (5), 2003. 456-480.

Biocca, F., Harms, C. and Gregg, J., The Networked Minds Measure of Social Presence: Pilot Test of the Factor Structure and Concurrent Validity. 4th annual International Workshop on Presence, ISPR. 2001. pp. 1-9.

Bradley, M.M. and Lang, P. J., 'Measuring emotion: The Self-Assessment Manikin and the Semantic Differential'. Journal of Behavior Therapy and Experimental Psychology, 25(1), 2002. pp. 49-59.

Brave, S. and Nass, C. 'Emotion in Human-Computer Interaction' In Jacko, J. and Sears, A. (Editors) Handbook of Human-Computer Interaction. pp. 251217. Lawrence Erlbaum Associates, 2002.

Bradner, E. and Mark, G., Social presence with video and application sharing. In Proc. of the 2001 International ACM Conference on Supporting Group Work, ACM, USA. 2001. pp 154-161.
Connell, J., Mendelsohn, G., Robins, R. and Canny, J., Effects of communication medium on interpersonal perceptions. GROUP '01: Proceedings of the 2001 International ACM SIGGROUP Conference on Supporting Group Work, ACM Press: New York. 2001. pp 117-124.

Frijda, N.H., Varieties of affect: Emotions and episodes, moods and sentiments. In P. Ekman and R.J. Davidson, editors, The nature of emotion, pages 59-67. Oxford University Press, New York, 1994.

Gooch, D. and Watts, L., 'The Magic Sock Drawer Project' Ext. Abstracts, CHI 2011, pp 243 - 252.

Hauber, J., Regenbrecht, H., Billinghurst, M. and Cockburn, A., Spatiality in videoconferencing: tradeoffs between efficiency and social presence. CSCW '06: Proceedings of the 2006 20th anniversary conference on Computer supported cooperative work, ACM Press: New York. 2006. pp. 413-422.

Kaye, J., I just clicked to say I love you: rich evaluations of minimal communication. In $\mathrm{CHI}$ ' 06 extended abstracts on Human factors in computing systems. ACM, USA, 2006. pp. 363-368.

Pace, T., Bardzell, S. and Bardzell, J., 'The rogue in the lovely black dress: intimacy in world of warcraft'. In Proceedings of the 28th International Conference on Human factors in Computing Systems (CHI'10). ACM, New York, NY, USA, 2010. pp. 233-242.

Riche, Y., Riche, N., Isenberg, P. and Bezerianos, A., Hard-to-use interfaces considered beneficial (some of the time). In Extended Abstracts of the 28th International Conf. on Human factors in Computing Systems. ACM, USA, 2010. pp. 2705-2714.

Shih, L. and Swan, K., Fostering social presence in asynchronous online class discussions. In Proc. of the 2005 conference on Computer support for collaborative learning, International Society of the Learning Sciences. 2005. pp. 602-606.

Short, J., Williams, E. and Christie, B., The Social Psychology of Telecommunications. John Wiley \& Sons, London, UK. 1976.

Tollmar, K. and Persson, J., Understanding remote presence. In Proc. of the second Nordic conference on Human-computer interaction. ACM, New York, USA, 2002. pp 41-50.

Tsujita, H., Tsukada, K. and Siio, I., SyncDecor: communication appliances for virtual cohabitation. In Proc. of the working conference on Advanced visual interfaces. ACM, USA, 2008. pp. 449-453.

Van Baren, J. and IJsselsteijn, W., Measuring Presence: A Guide to Current Measurement Approaches. Technical Report, Deliverable of the OmniPres project IST-2001-39237, number 5. 2004.

Walther, J.B., Computer-mediated communication: Impersonal, interpersonal, and hyperpersonal interaction. Communication Research, 23 (1). 3-43. 